\title{
Neues aus der Schlafmedizin für Neurologen und Psychiater
}

\author{
Aus einer Vielzahl von Vorträgen, die der Kongress der Deutschen Gesellschaft für Schlaffor- \\ schung und Schlafmedizin (DGSM) seinen Besuchern vom 9. bis 11. November 2017 bot, stachen \\ einige besonders heraus: Darunter Sex im Schlaf und die nicht immer einfache Differenzial- \\ diagnose der zugrunde liegenden Störungen. Auch RLS und Änderungen der Diagnosekriterien \\ sowie die Früherkennung der Augmentation und therapeutische Optionen für Schlaganfallpati- \\ enten mit Atmungsstörungen waren Thema der Tagung.
}

\section{Schlaf und Schlaganfall: CPAP präventiv sinnvoll}

Noch sind die Daten zur Reduktion von Morbidität und Mortalität durch CPAP limitiert - aktuelle Studienergebnisse lassen den Einsatz bei Schlaganfallpatienten mit Atmungsstörungen jedoch sinnvoll erscheinen.

Schlafbezogene Atmungsstörungen treten nach einem Schlaganfall gehäuft auf und haben negative Folgen auf das kognitive Outcome und auf die Erholung der Patienten. Etwa 50 - 70\% sind betroffen, die Häufigkeit ist bei transitorischer ischämischer Attacke (TIA) und manifestem Schlaganfall vergleichbar, eine territoriale Präferenz wurde bislang nicht festgestellt. Die Folgen sind schwerwiegend, wie Professorin Svenja Happe, Chefärztin der Neurologie an der Klinik Maria Frieden, Teltge, berichtete. Die 10-Jahresmortalität ist gesteigert [Sahlin C et al. Arch Intern Med 2008; 168 (3): 297 - 301], die funktionale Erholung reduziert und die Hospitalisierungsdauer verlängert, Delir und Depression erhöhen die Morbidität, kognitive Defizite treten häufig auf. Ein Screening auf Atmungsstörungen bei Schlaganfallpatienten sei daher sinnvoll.

Therapeutisch ist neben Gewichtsabnahme und Verbesserung der Schlafhygiene die CPAP-Beatmung hilfreich, wie eine randomisierte kontrollierte Studie gezeigt hat [Bravata DM et al.
Sleep 2011; 34 (9): 1271 - 7]. Dabei erhielten 31 von 55 Patienten mit Schlaganfall in der Akutphase eine autoadjustierte CPAPBeatmung in den ersten 30 Tagen. Die Ergebnisse: Die Adhärenz war mit $100 \%$ gut $(63 \%>4$ Stunden, $37 \%<4$ Stunden). Das Outcome war mit einer Verbesserung des Scores auf der NIHSS (National Institutes of Health Stroke Scale) gegenüber der Kontrollgruppe signifikant besser $(p=0,03)$, vaskuläre Ereignisse traten seltener auf. Auch für den präventiven Einsatz der CPAP-Beatmung gibt es positive Daten [Martinez-Garcia MA et al. Am J Respir Crit Care Med 2012; 186 (9): 909 - 16]: So war unter der Beatmung die kumulative Mortalität bei älteren Patienten mit obstruktivem Schlafapnoesyndrom (OSAS) nahezu auf dem Niveau gesunder Kontrollen, während sie sich bei OSAS-Patienten ohne Beatmung über ein Follow-up von bis zu zehn Jahren deutlich steigerte.

Eine europäische Leitlinie zu Schlaf und Schlaganfall unter der Beteiligung verschiedener europäischer Fachgesellschaften ist derzeit noch in Arbeit und könnte 2018 veröffentlicht werden.

Michael Koczorek

Symposium „Europäische Leitlinien der neurologischen Schlafmedizin - ein Update", 25. Kongress der Deutschen Gesellschaft für Schlafforschung und Schlafmedizin, Münster, 9.11.2017 